\title{
Subnicho etario y biología reproductiva de Parachondria neglectus (Littorinimorpha: Annulariidae) en Manzanillo, Cuba
}

\author{
Sandra Sariego Frómeta ${ }^{*}$, Orlando Ramón Sariego Tamayo ${ }^{2}$, Jorge Erick Marin Morán ${ }^{3}$ \\ \& Luiz Ricardo L. Simone ${ }^{1}$ \\ 1. Laboratorio de Malacología. Museo de Zoología de la Universidad de São Paulo (MZUSP). São Paulo, Brasil; \\ sandrasariego@usp.br, lrsimone@usp.br \\ 2. Departamento de Ingeniería Forestal. Facultad de Ciencias Agrícolas. Universidad de Granma. Bayamo, Cuba; \\ osariegot@udg.co.cu \\ 3. Escuela de Ingeniería de São Carlos / Universidad de São Paulo (EESC/USP). São Carlos, Brasil; \\ jmarin74@yahoo.es \\ * Correspondencia
}

Recibido 20-II-2018. Corregido 06-VIII-2018. Aceptado 26-IX-2018.

\begin{abstract}
Age-related subniche and reproductive biology of Parachondria neglectus (Littorinimorpha: Annulariidae) in Manzanillo, Cuba. Parachondria neglectus is a land snail of the Annulariidae family, endemic to Cuba and microlocalized in Granma province. In the present research, the temporal dynamics of age-related subniche and some aspects of the reproductive biology of a P. neglectus population, located in ManzanilloGranma-Cuba, were characterized. Thirty expeditions were performed from December 2013 to February 2015. The comparisons between the length $(\mathrm{t}=8.05, \mathrm{P}=0.007)$ and biggest diameter $(\mathrm{t}=6.24, \mathrm{P}=0.0001)$ of the shells of truncated juveniles and the shells of full juveniles showed significant statistical differences, indicating that both groups represent two kinds of different ages. The comparison between the biggest diameter of the shells of truncated adults and the shells of full adults $(\mathrm{t}=1.50, \mathrm{P}=0.16)$ showed that both groups represent the same kind of age in the population. The existence of significant statistical differences between the length $(t=19.45$, $\mathrm{P}=0.0003)$ and the biggest diameter $(\mathrm{t}=13.19, \mathrm{P}=0.006)$ of the females' shells and the males' shells verify the sexual dimorphism in the species, proving to be the females of bigger dimensions in relation to the males. The whitish circular spot in the shells is a valid external morphological characteristic to differentiate the adult females already mated in previous occasions from the rest of the members of the population. The reproductive period was from July to December 2014. Copulations were observed from July to October 2014 and they were associated to conditions of abundant precipitations and high temperatures of summer. The recruitment period took place from September to December 2014.
\end{abstract}

Key words: land snails; Parachondria neglectus; ecological niche; reproduction.

Sariego Frómeta, S., Sariego Tamayo, O. R., Marin Morán, J. E., \& Simone, L. R. L. (2018). Subnicho etario y biología reproductiva de Parachondria neglectus (Littorinimorpha: Annulariidae) en Manzanillo, Cuba. Revista de Biología Tropical, 66(4), 1664-1673.

La malacofauna terrestre de Cuba está reconocida entre las más diversas del mundo con 1392 especies inventariadas y un endemismo del 96 \% (Hernández et al., 2017). Neritimorpha y Caenogastropoda en Cuba incluyen 476 especies terrestres agrupadas en 6 familias.
La familia más extensa es Annulariidae que contiene 365 especies ordenadas en 35 géneros (31 endémicos) (Espinosa \& Ortea, 2009). Parachondria (Dall, 1905) es el género de más amplia distribución dentro de esta familia en Cuba e incluye especies muy microlocalizadas, 
lo que las hace especialmente vulnerables. Tal es el caso de Parachondria (Parachondria) neglectus (Pfeiffer, 1858), endémica y microlocalizada en la provincia de Granma.

La combinación taxonómica más actualizada fue propuesta por Watters (2006) en una revisión de taxa de annuláridos de América Central. Pero la especie también ya fue considerada de los géneros Cyclostoma y Chondropoma, y tiene como sinónimo Chondropoma revinctum (Poey in Pfeiffer, 1858).

Se estima que al menos entre 132 y 260 especies de moluscos terrestres cubanos actualmente presentan algún grado de amenaza. Sin embargo, estas cifras parecen alejadas de la situación real. Desafortunadamente, según la Lista roja de la IUCN (2008) ningún molusco terrestre del archipiélago cubano está incorporado en alguna categoría de amenaza, lo cual dificulta la obtención de financiamiento para la investigación, conservación y educación ambiental con fines de protección (Maceira \& Espinosa, 2016).

$P$. neglectus no está incluida de forma explícita en la Lista roja de la fauna cubana (Amaro-Valdés, 2012), ni en el Libro rojo de invertebrados terrestres de Cuba (Maceira \& Espinosa, 2016), a pesar de que su extensión de presencia es de $1091 \mathrm{~km}^{2}$ (Sariego, 2006). Por lo que, de acuerdo con los criterios de IUCN (2012), debería ser considerada como amenazada de extinción y valorarse la categoría de "en peligro" (EN) siguiendo el criterio B que se refiere a una extensión de presencia menor de $5000 \mathrm{~km}^{2}$, areal fragmentado y declinación continua observada o inferida de la extensión de presencia, del área de ocupación y de la calidad del hábitat.

Reportes recientes señalan que de los 8113 $\mathrm{km}^{2}$ de superficie territorial sin aguas interiores de Granma, las cuales representan el $7.8 \%$ del total estimado para Cuba, solo el $26.6 \%$ está cubierto de bosques naturales (ONEI, 2017c). Estos datos reafirman que el territorio que constituía el ámbito original de esta especie ha sido severamente impactado por las actividades humanas.
Lo anterior, añadido al hecho de involucrarse localidades del ámbito de distribución de la especie dentro o muy cerca del Parque Nacional Desembarco del Granma implicó la conveniencia de abordar esta investigación con fines conservacionistas.

\section{MATERIALES Y MÉTODOS}

Área de estudio: El municipio de Manzanillo ubicado en la provincia de Granma, Cuba está situado entre $\left(20^{\circ} 03^{\prime}-20^{\circ} 26^{\prime} \mathrm{N} \& 77^{\circ} 14^{\prime}\right.$ - $\left.77^{\circ} 07^{\prime} \mathrm{W}\right)$. Posee una extensión superficial de $498.95 \mathrm{~km}^{2}$ (ONEI, 2017a) y se encuentra en la llanura del Cauto-Guacanayabo. Su paisaje está definido como colinas carsificadas, con pastos y matorrales secundarios (Mateo, 1989). Los promedios de temperaturas mínimas y máximas anuales son de 21.1 y $31.6{ }^{\circ} \mathrm{C}$. Se registran un promedio de 100 días lluviosos al año y la precipitación media anual es de $1045.2 \mathrm{~mm}$ (ONEI, 2017b). La humedad relativa se mantiene elevada durante todo el año con un promedio del $76 \%$ (ONEI, 2017a). La localidad estudiada se encuentra en el poblado Blanquizal a $18 \mathrm{msnm}$. Se puede definir como una formación vegetal arbórea, fuertemente antropizada por prácticas relacionadas con la ganadería, tala y podas.

Para caracterizar la dinámica temporal del subnicho etario y la biología reproductiva de la población de $P$. neglectus se seleccionaron parcelas de $3 \times 3 \mathrm{~m}$. La cantidad de moluscos analizados osciló entre 160 y 385 por mes. Se efectuaron 2 muestreos mensuales en el período comprendido entre diciembre 2013 y febrero 2015. Para estimar el tamaño de los individuos fue utilizada una regla graduada LEICA 10310345 (de precisión $0.1 \mathrm{~mm}$ ) con la que fueron medidos el largo y el diámetro mayor de la concha. El largo fue medido desde el ápice de la concha hasta el borde del labio en los ejemplares que estaban completos y desde el borde del labio hasta el extremo más largo de la truncadura, en los que habían perdido la porción apical de la concha. Los valores de los milímetros de lluvia acumulados en el período de estudio se obtuvieron de la Estación 
Meteorológica de Manzanillo publicados por ONEI (2017a).

Subnicho etario de $\boldsymbol{P}$. neglectus: La terminología utilizada para el estudio del subnicho etario es la sugerida por Silva y Berovides (1982) teniendo en cuenta las subdivisiones propuestas por Berovides, Genaro y Sánchez (1988). La presencia del peristoma replegado permitió la identificación de los adultos.

Biología reproductiva de $P$. neglectus: La observación de las cópulas se realizó en condiciones de laboratorio. Se seleccionaron individuos que formaron parejas en condiciones naturales los cuales fueron colocados en terrarios $(40 \times 30 \times 25 \mathrm{~cm})$. El fondo se cubrió con una capa de tierra de $4 \mathrm{~cm}$ de espesor. El alimento suministrado consistió en hojas y ramas infestadas de hongos. La temperatura se mantuvo entre 22 y $28{ }^{\circ} \mathrm{C}$. El ciclo de iluminación se estableció según la razón de horas de luz y oscuridad (12:12). Los recipientes de experimentación fueron revisados diariamente para buscar signos de actividad reproductiva: cópulas, nidos frescos y/o eclosiones. La observación del pene de los machos y su posterior intromisión en las hembras durante la cópula, además de la disección de ejemplares adultos, permitió la identificación entre sexos. En los moluscos que utilizaron el tronco como sustrato de apareamiento, la altura sobre el suelo fue medida con una cinta métrica. Para determinar la orientación geográfica de los moluscos que formaron parejas en la localidad se utilizó una brújula BRUNTON B20.

A través del cálculo de la media, la desviación estándar y el coeficiente de variación se caracterizaron las variables conquiológicas procesadas estadísticamente. La prueba $t$ de Student fue utilizada para comparar las medias del largo y del diámetro mayor de las conchas de los juveniles y los adultos truncados y completos, así como las medias del largo y del diámetro mayor de las conchas de las hembras y los machos. Se efectuó una prueba de Independencia con la realización de una tabla de contingencia 2 × 2 con corrección de Yates para conocer si la ocurrencia de las precipitaciones influía en la frecuencia de utilización de los diferentes sustratos en los adultos. Todos los cálculos estadísticos se realizaron a través del programa STATISTICA 13.0 (Statsoft, 2015).

\section{RESULTADOS}

Subnicho etario de $P$. neglectus: Del total de observaciones realizadas durante el período evaluado, la población estudiada de $P$. neglectus estuvo constituida por el $24.71 \%$ de juveniles y el $75.29 \%$ de adultos. A excepción de octubre 2014, en los restantes meses muestreados los adultos fueron más numerosos que los juveniles. Los juveniles conformaron del 36.10 al $56.83 \%$ de la población entre septiembre y diciembre 2014, indicando que en este período se efectuó el reclutamiento en la población. De junio a agosto 2014 se encontraron los mayores porcentajes de adultos con valores del 88.33 al 92.98 \% (Fig. 1). Las mediciones conquiológicas realizadas presentaron mayores coeficientes de variación en los juveniles para las dos variables analizadas (Cuadro 1).

Las mediciones mensuales del diámetro mayor de las conchas de los moluscos confirmaron que en octubre 2014 ocurrió el pico del período de reclutamiento con un valor promedio de $4.38 \pm 0.90 \mathrm{~mm}$. En julio 2014 se observó el valor promedio más elevado del diámetro de las conchas $(5.44 \pm 0.51 \mathrm{~mm})$, correspondiéndose con el período en el que se encontró la mayor cantidad de adultos en la población (Cuadro 2).

Las comparaciones del largo $(\mathrm{t}=8.05$, $\mathrm{P}=0.007)$ y del diámetro mayor $(\mathrm{t}=6.24$, $\mathrm{P}=0.0001$ ) de los juveniles truncados y los juveniles completos mostraron diferencias estadísticas significativas, sugiriendo que ambos grupos representan dos clases de edades diferentes en la población. Los valores medios del largo de los adultos truncados y de los adultos completos fueron estadísticamente diferentes $(\mathrm{t}=4.82, \mathrm{P}=0.002)$. Sin embargo, la comparación del diámetro mayor de sus conchas $(\mathrm{t}=1.50, \mathrm{P}=0.16)$ demostró que conforman una misma clase de edad. La diferencia de las 


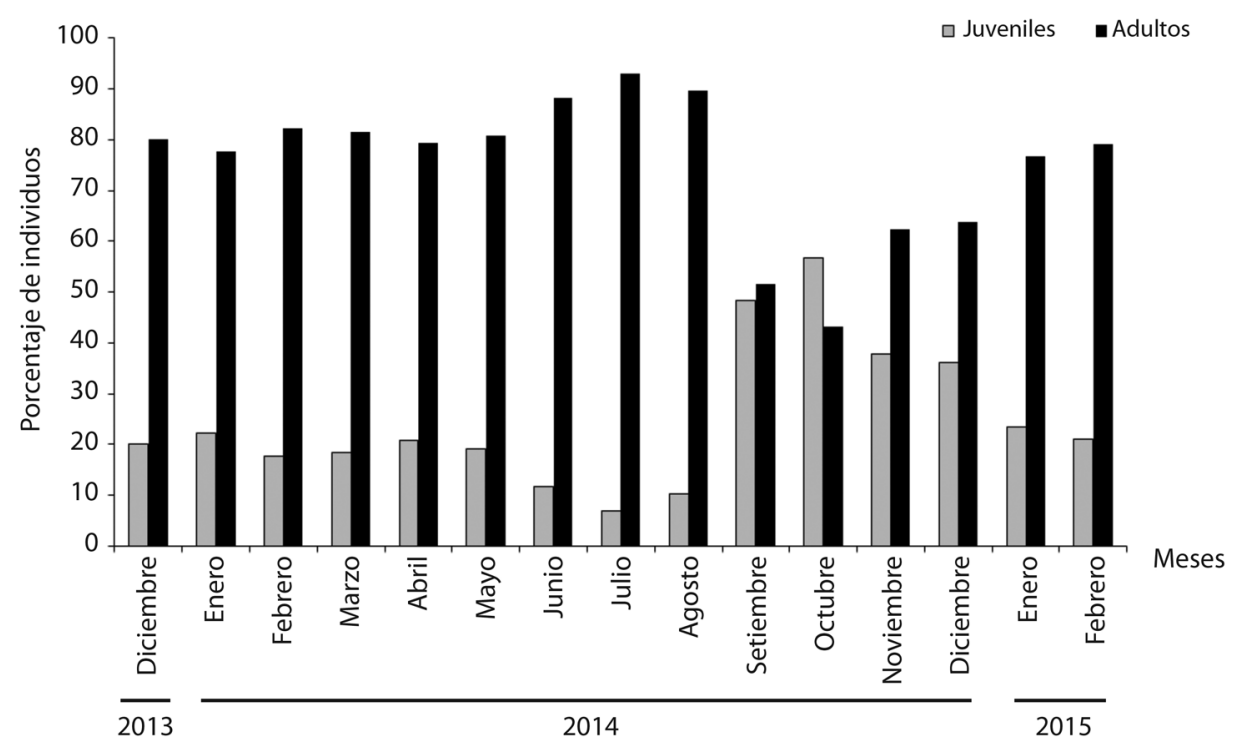

Fig. 1. Variación temporal del porcentaje de individuos por grupos etarios de la población estudiada de P. neglectus en Manzanillo.

Fig. 1. Temporal variation of the percentage of individuals by age groups of the studied population of $P$. neglectus in Manzanillo.

\section{CUADRO 1}

Mediciones conquiológicas por grupos etarios de P. neglectus en Manzanillo

TABLE 1

Conchological measurements by age groups of $P$. neglectus in Manzanillo

\begin{tabular}{llcccc}
\multicolumn{2}{c}{ Grupos etariós } & $\overline{\mathrm{X}} \pm \mathrm{DS}$ & $\mathrm{CV}(\%)$ & $\overline{\mathrm{X}} \pm \mathrm{DS}$ & \multicolumn{2}{c}{ CV $(\%)$} \\
\multirow{2}{*}{ Juvenímetro mayor $(\mathrm{mm})$} \\
\multirow{3}{*}{ Adultos } & Completos & $8.31 \pm 2.16$ & 25.99 & $3.24 \pm 0.64$ & 19.75 \\
& Truncados & $11.55 \pm 2.56$ & 22.16 & $4.95 \pm 0.93$ & 18.79 \\
& Completos & $14.07 \pm 1.42$ & 10.09 & $5.43 \pm 0.51$ & 9.39 \\
\hline
\end{tabular}

X: Media; DS: Desviación estándar; CV: Coeficiente de variación.

medias del largo de los adultos completos y de los adultos truncados apuntó que este grupo etario pierde aproximadamente $0.81 \mathrm{~mm}$ del ápice de la concha (Cuadro 1).

Del total de observaciones realizadas el $71.64 \%$ de los moluscos presentaban las conchas truncadas. En octubre 2014 los juveniles completos (que representan la menor clase de edad) conformaron el $50 \%$ de la población. Los porcentajes mensuales de los juveniles truncados y de los adultos completos no superaron el $11 \%$ en el período de estudio. De junio a agosto 2014 se detectaron los mayores porcentajes de adultos truncados en la población, con valores del 83 al $90 \%$ (Fig. 2).

Biología reproductiva de $\boldsymbol{P}$. neglectus: En 10 de los 15 meses muestreados se observaron parejas, para un total de 133. El mayor número de parejas se detectó en julio y en agosto 2014 
CUADRO 2

Variación temporal del diámetro de las conchas $(\mathrm{mm})$ de la población estudiada de P. neglectus en Manzanillo

TABLE 2

Temporal variation the diameter of shells $(\mathrm{mm})$ of the studied population of P. neglectus in Manzanillo

\begin{tabular}{lcc}
\multicolumn{1}{c}{ Meses } & \multicolumn{2}{c}{ Diámetro mayor $(\mathrm{mm})$} \\
Diciembre 2013 & $5.23 \pm 0.66$ & $\mathrm{CV}(\%)$ \\
Enero 2014 & $5.22 \pm 0.69$ & 12.62 \\
Febrero 2014 & $5.26 \pm 0.61$ & 13.22 \\
Marzo 2014 & $5.32 \pm 0.63$ & 11.60 \\
Abril 2014 & $5.18 \pm 0.56$ & 11.84 \\
Mayo 2014 & $5.27 \pm 0.58$ & 10.81 \\
Junio 2014 & $5.33 \pm 0.55$ & 11.01 \\
Julio 2014 & $5.44 \pm 0.51$ & 10.32 \\
Agosto 2014 & $5.34 \pm 0.53$ & 9.38 \\
Septiembre 2014 & $4.45 \pm 0.79$ & 9.93 \\
Octubre 2014 & $4.38 \pm 0.90$ & 17.75 \\
Noviembre 2014 & $4.82 \pm 0.77$ & 20.55 \\
Diciembre 2014 & $4.96 \pm 0.74$ & 15.98 \\
Enero 2015 & $5.08 \pm 0.70$ & 14.92 \\
Febrero 2015 & $5.21 \pm 0.68$ & 13.78 \\
\hline
\end{tabular}

$\bar{X}$ : Media; DS: Desviación estándar; CV: Coeficiente de variación.

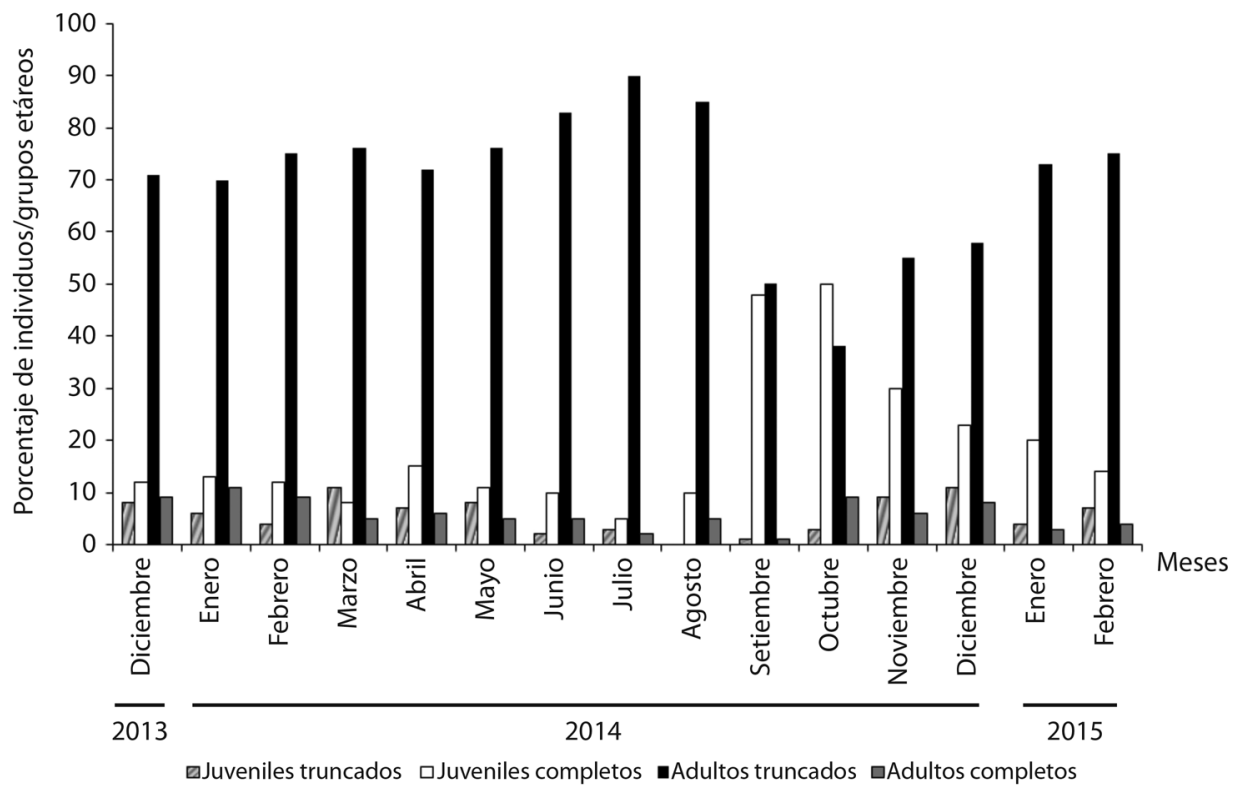

Fig. 2. Variación temporal de los porcentajes de juveniles truncados y completos y de los adultos truncados y completos en la población estudiada de $P$. neglectus en Manzanillo.

Fig. 2. Temporal variation of the percentages of truncated and full juveniles and of truncated and full adults in the studied population of $P$. neglectus in Manzanillo. 
(con 37 y 31 respectivamente). No se encontraron parejas en febrero, abril y noviembre 2014, además de enero y febrero 2015.

De acuerdo a la clasificación de Espinosa y Ortea (2009), P. neglectus es un molusco terrícola asociado a la vegetación. No obstante, la prueba de independencia con corrección de Yates demostró que de manera general en los adultos $\left(\mathrm{X}^{2}=117.34, \mathrm{P}=0.0001\right)$ la frecuencia de utilización de un sustrato u otro depende de la ocurrencia de las precipitaciones. Del análisis de la figura 3 es posible plantear que el tronco de los arbustos y árboles constituye en la localidad estudiada el sustrato preferencial de esta especie para su reproducción (Fig. 3). La altura sobre el suelo de las parejas que emplearon el tronco como sustrato presentó un valor medio de $165.62 \pm 61.27 \mathrm{~cm}$.

De manera general el $46.60 \%$ de las parejas que utilizaron el tronco como sustrato se orientaron hacia el norte, el $32.75 \%$ hacia el sur, el $13.67 \%$ hacia el oeste y el $6.98 \%$ hacia el este.

Los análisis realizados en el laboratorio teniendo en cuenta las disecciones de ejemplares indicaron que las hembras representan aproximadamente el $69.30 \%$ de la población.
Dimorfismo sexual: La existencia de diferencias estadísticas significativas entre el largo $(\mathrm{t}=19.45, \mathrm{P}=0.0003)$ y el diámetro mayor $(\mathrm{t}$ $=13.19, \mathrm{P}=0.006)$ de las conchas de las hembras y los machos confirmaron el dimorfismo sexual en la especie, resultando las hembras de mayores dimensiones respecto a los machos (Cuadro 3, Fig. 4).

La disección de ejemplares con mancha circular blancuzca en las conchas ratificó que en su totalidad eran hembras. La mancha circular se observó con nitidez, presentó bordes bien marcados, como indicando una unión, con contracción del pie del macho, fuerte y prolongada. $\mathrm{Su}$ forma coincidió con la forma de la apertura bucal de las conchas de los machos (Fig. 4).

En el $77.44 \%$ de las parejas observadas en la localidad uno de los individuos presentaba la mancha circular.

Cópula: En condiciones de laboratorio y en la localidad estudiada las cópulas ocurrieron de julio a octubre 2014. Durante la cópula, el macho ubicado encima de la hembra permanecía fuertemente adherido por el pie al cuadrante que forman el labio y la porción final de la sutura de la última vuelta de la concha de la

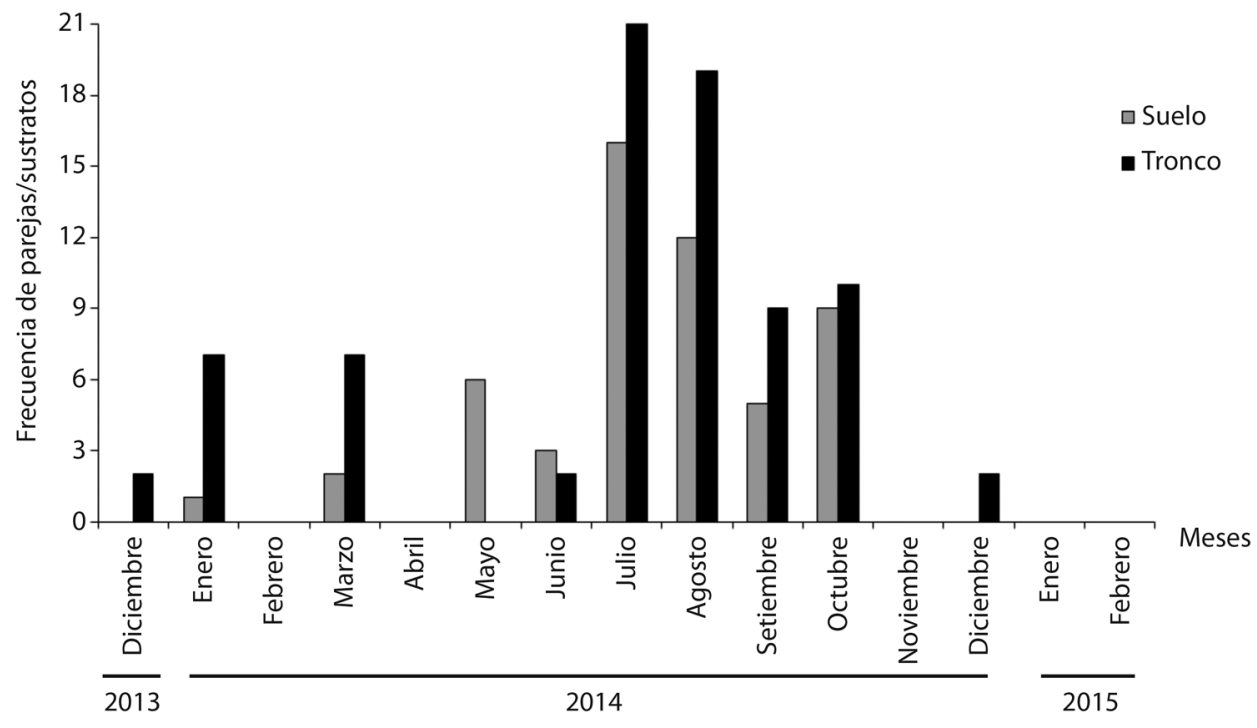

Fig. 3. Variación temporal de la frecuencia de parejas por sustratos en la población estudiada de P. neglectus en Manzanillo. Fig. 3. Temporal variation of the frequency of sexual partners by substrates in the studied population of $P$. neglectus in Manzanillo. 
CUADRO 3

Mediciones conquiológicas de las hembras y los machos de la población estudiada de P. neglectus en Manzanillo

TABLE 3

Conchological measurements of females and males of the studied population of $P$. neglectus in Manzanillo

\begin{tabular}{|c|c|c|c|c|c|c|c|c|}
\hline \multirow{2}{*}{ Sexos } & \multicolumn{4}{|c|}{ Largo (mm) } & \multicolumn{4}{|c|}{ Diámetro mayor (mm) } \\
\hline & $\overline{\mathrm{X}} \pm \mathrm{DS}$ & CV $(\%)$ & $\operatorname{Rec}$ & ido & $\overline{\mathrm{X}} \pm \mathrm{DS}$ & $\mathrm{CV}(\%)$ & & \\
\hline Hembras & $14.02 \pm 0.77$ & 5.51 & 12.20 & 16.30 & $5.79 \pm 0.32$ & 5.56 & 5.10 & 6.80 \\
\hline Machos & $12.01 \pm 0.82$ & 6.86 & 9.10 & 13.50 & $5.20 \pm 0.38$ & 7.28 & 4.70 & 6.40 \\
\hline
\end{tabular}

$\bar{X}$ : Media; DS: Desviación estándar; CV: Coeficiente de variación.
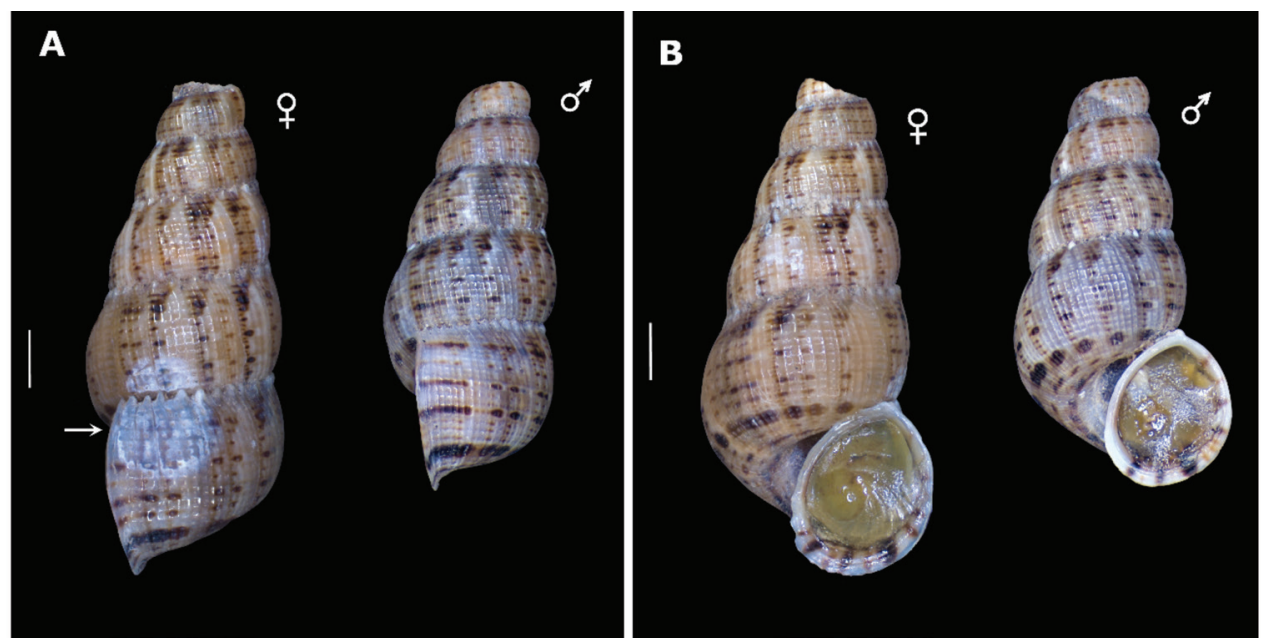

Fig. 4. Dimorfismo sexual en $P$. neglectus. A. Vista derecha de las conchas de una hembra con mancha circular blancuzca (flecha) y un macho de P. neglectus. B. Vista apertural de las conchas de una hembra y un macho de P. neglectus. Escala $=2 \mathrm{~mm}$.

Fig. 4. Sexual dimorphism in P. neglectus. A. Right view of the shells of a female with a whitish circular spot (arrow) and a male of $P$. neglectus. B. Apertural view of the shells of a female and a male of $P$. neglectus. Scale $=2 \mathrm{~mm}$.

hembra. Luego, con el opérculo semiabierto proyectaba el pene detrás del tentáculo derecho. En P. neglectus el pene es largo y ancho, romboidal en el extremo y aplanado. En los ejemplares observados el pene alcanzó valores medios de $5.24 \mathrm{~mm}$ de largo y $1.5 \mathrm{~mm}$ de ancho. Este órgano era utilizado por el macho primeramente para estimular repetidamente el opérculo de la hembra a iniciar su apertura. La hembra era penetrada con el opérculo prácticamente cerrado. Durante la cópula, ambos individuos (hembra y macho) permanecieron cubiertos por una secreción mucosa.

\section{DISCUSIÓN}

Durante el período de estudio la estructura poblacional de $P$. neglectus fue caracterizada por la presencia de por lo menos dos clases etáreas juveniles y una adulta. La existencia de adultos en mayor proporción que los juveniles ha sido registrado también para poblaciones de otras especies cubanas como Polymita sulphurosa (Fernández, González, \& Reyes, 1998), Zachrysia guanensis costulata (Tadeo, Alfonso, \& Berovides, 1999) y Caracolus sagemon (Maceira, 2010). 
Debido a que estos moluscos se truncan, la medición del diámetro de las conchas constituye el criterio adecuado para determinar la dinámica de los tamaños en sus poblaciones. La pérdida de la región apical de las conchas en especies de esta familia de prosobranquios se ha atribuido a razones adaptativas, como dejar de cargar una parte vacía de la concha. Se ha planteado además, que sucede como consecuencia de una estrategia defensiva de estos moluscos al arrojarse de forma voluntaria de árboles y paredones ante la presencia de un depredador (Espinosa \& Ortea, 2009).

Ninguno de los ejemplares muestreados superó los $6.8 \mathrm{~mm}$ de diámetro mayor reportado para el ejemplar tipo de $P$. neglectus recolectado en Cabo Cruz y descrito por Torre y Bartsch (1938).

En otras especies de prosobranquios se ha reportado que las hembras son más numerosas que los machos con porcentajes del $80 \%$ en Bithynia leachi, del $67 \%$ en Littorina littorea y del $63 \%$ en Rissoa parva y Turritella communis (Pelseneer, 1926). Para especies de los géneros Littorina (Moore, 1937), Strombus (Abbott, 1960), Oncomelania (Chi \& Wagner, 1957) y Tudorella (Ibañez \& Alonso, 1979) se ha informado la existencia de dimorfismo sexual. Estas diferencias de tamaño han sido atribuidas a tasas de crecimiento desiguales, presentando las hembras mayores dimensiones conquiológicas (Giese \& Pearse, 1977). La presencia de un pene grande respecto a las dimensiones corporales se ha descrito en Annulariidae. En Meganipha rhecta es delgado, triangular en la sección transversal y de origen paleal (Thompson, 1978). En Annularia sp. es curvado en la base y en el ápice, ligeramente aplastado y de longitud similar a la de la cavidad paleal (Simone, 2004).

Las disecciones realizadas corroboraron que, como resultado del acto de apareamiento, los machos dejan una mancha circular blancuzca en las conchas de las hembras en el lugar en el que se colocaron durante la cópula. Por tanto, constituye una característica morfológica externa válida para diferenciar a las hembras adultas y ya apareadas en ocasiones anteriores, del resto de los miembros de la población.

La observación de 31 tríos de julio a octubre 2014 sugiere la posibilidad de competencia reproductiva entre los machos por las hembras maduras sexualmente (selección intrasexual).

Teniendo en cuenta que los vientos predominantes en Manzanillo son del Este y tienen una velocidad promedio anual de $9.1 \mathrm{~km} / \mathrm{h}$ (ONEI, 2017a), es posible asumir que en la localidad los vientos no ejercen influencias perjudiciales para la especie. La preferencia de las parejas por las orientaciones geográficas en dirección al Norte posiblemente constituye un mecanismo adoptado para evitar las radiaciones solares intensas.

En $P$. neglectus como en la mayoría de los gastrópodos prosobranquios, la actividad gamética y sincrónica dentro de las poblaciones y la maduración sexual acontece en un determinado período (Ilano, Fujinaga, \& Nakao, 2003). Las cópulas estuvieron asociadas a condiciones de precipitaciones abundantes y temperaturas altas del verano. Se ha informado que el desarrollo estacional de las gónadas en los moluscos terrestres está relacionado con las altas temperaturas (Albrecht, Koch, Carreño, \& Castro-Vazquez, 2005). En el período de estudio se registraron en Manzanillo acumulados de $1110 \mathrm{~mm}$ de lluvia, de los cuales $467 \mathrm{~mm}$ se correspondieron de julio a octubre de 2014 (ONEI, 2017a). En tres elevaciones cársticas del complejo cubano de vegetación de mogote Escaleras de Jaruco, las precipitaciones constituyeron una de las variables climáticas más importantes para el desarrollo de especies de Annulariidae entre las que se encuentran: Rhytidopoma nodulatum, Torrella inmersa y Chondropoma pictum (Hernández, 2013; Hernández \& Reyes-Tur, 2013).

No se encontraron huevos de P. neglectus; sin embargo, se observaron moluscos recién nacidos en condiciones de laboratorio y en la localidad estudiada. Lo anterior pudiera sugerir que en esta especie como en otras especies de gastrópodos, se evidencia el fenómeno de tigmotaxis ocurriendo las puestas en oquedades 
(Fernández \& Lajonchere, 2013) debajo de las piedras y entre la hojarasca.

Los resultados de la presente investigación aportan nuevos conocimientos de la ecología de esta especie endémica de Cuba y microlocalizada en la provincia de Granma y podrían resultar de gran utilidad en los futuros planes de manejo de P. neglectus.

Declaración de ética: los autores declaran que todos están de acuerdo con esta publicación y que han hecho aportes que justifican su autoría; que no hay conflicto de interés de cualquier tipo; y que han cumplido con todos los requisitos y procedimientos éticos y legales pertinentes. El documento firmado se encuentra en los archivos de la revista.

\section{AGRADECIMIENTOS}

Este trabajo contó con el apoyo del Laboratorio de Malacología del Museo de Zoología de la Universidad de São Paulo (MZUSP). Se agradece también a la Fundación de Amparo a la Pesquisa del Estado de São Paulo (FAPESP) por la beca otorgada y por el financiamiento asignado al proceso: 2016/13231-9.

\section{RESUMEN}

Parachondria neglectus es un molusco terrestre de la familia Annulariidae, endémico de Cuba y microlocalizado en la provincia de Granma. En la presente investigación se caracterizaron la dinámica temporal del subnicho etario y algunos aspectos de la biología reproductiva de una población de P. neglectus localizada en Manzanillo, GranmaCuba. Se realizaron 30 expediciones entre diciembre 2013 y febrero 2015. Las comparaciones del largo $(t=8.05, P=$ $0.007)$ y diámetro mayor $(\mathrm{t}=6.24, \mathrm{P}=0.0001)$ de las conchas de los juveniles truncados y los juveniles completos mostraron diferencias estadísticas significativas, indicando que ambos grupos representan dos clases de edades diferentes. La comparación del diámetro mayor de las conchas de los adultos truncados y los adultos completos $(\mathrm{t}=1.50$, $\mathrm{P}=0.16$ ) demostró que conforman un misma clase de edad en la población. La existencia de diferencias estadísticas significativas entre el largo $(t=19.45, \mathrm{P}=0.0003)$ y el diámetro mayor $(\mathrm{t}=13.19, \mathrm{P}=0.006)$ de las conchas de las hembras y los machos reafirmaron el dimorfismo sexual en la especie, resultando las hembras de mayores dimensiones respecto a los machos. La mancha circular blancuzca en las conchas es una característica morfológica externa válida para diferenciar a las hembras adultas y ya apareadas en ocasiones anteriores, del resto de los miembros de la población. El período reproductivo comprendió de julio a diciembre 2014. Las cópulas se observaron de julio a octubre 2014 y estuvieron asociadas a condiciones de precipitaciones abundantes y temperaturas altas del verano. El período de reclutamiento ocurrió de septiembre a diciembre 2014.

Palabras clave: caracoles terrestres; Parachondria neglectus; nicho ecológico; reproducción.

\section{REFERENCIAS}

Abbott, R. T. (1960). The genus Strombus in the IndoPacific. Indo-Pacific Mollusca, 1, 33-146.

Albrecht, E. A., Koch, E., Carreño, N. B., \& CastroVazquez, A. (2005). Control of the seasonal arrest of copulation and spawning in the apple snail Pomacea canaliculata (Prosobranchia: Ampullariidae): differential effects of food availability, water temperature, and day length. Veliger, 47(3), 169-174.

Amaro-Valdés, S. (2012). Lista roja de la fauna cubana. La Habana, Cuba: Editorial AMA.

Berovides, V., Genaro, J. A., \& Sánchez, C. S. (1988). Nuevas consideraciones acerca del nicho ecológico. Ciencias Biológicas, 19-20, 3-8.

Chi, L. W., \& Wagner, E. D. (1957). Studies on reproduction and growth of Oncomelania quadrasi, $O$. nosophora, and O. formosana, snail hosts of Schistosoma japonicum. The American Journal of Tropical Medicine and Hygiene, 6(5), 949-960.

Espinosa, J., \& Ortea, J. (2009). Moluscos terrestres de Cuba. Vasa, Finlandia: Spartacus-Säätiö- Spartacus Foundation y Sociedad Cubana de Zoología, UPC Print.

Fernández, A., González, A., \& Reyes, E. (1998). Population density of Polymita sulphurosa Morelet (Mollusca: Helminthoglyptidae) in the Yaguaneque Hill, Holguín Province, Cuba: A conservacionist alarm. Of Sea and Shore, 21(1), 49-52.

Fernández, A. \& Lajonchere, L. (2013). Reproducción de Liguus fasciatus (Mollusca: Orthalicidae): un caso de auto-fertilización en Cuba. Solenodon, 11, 82-87.

Giese, A. C., \& Pearse, J. S. (1977). Reproduction of Marine Invertebrates: Molluscs: Gastropods and Cephalopods (Vol. IV). New York-San Francisco \& London: Academic Press, Inc.

Hernández, M. (2013). Occupancy of strata of plant height and plant substrate by land snail Gastropoda 
assemblages at Escaleras de Jaruco, Mayabeque, Cuba. The Nautilus, 127(1), 29-35.

Hernández, M., Alvarez-Lajonchere, L., Martínez, D., Maceira, D., Fernández, A., \& Espinosa, J. (2017). Moluscos terrestres y dulceacuícolas. En C. A. Mancina \& D. D. Cruz. (Eds.), Diversidad biológica de Cuba: métodos de inventario, monitoreo y colecciones biológicas (pp. 168-195). La Habana, Cuba: Editorial AMA.

Hernández, M. \& Reyes-Tur, B. (2013). Composición y estructura en agregaciones de moluscos terrestres en el Complejo de vegetación de mogote, Escaleras de Jaruco, Cuba. Revista de Biología Tropical, 61(4), $1769-1783$

Ibañez, M. \& Alonso, M. R. (1979). Observaciones anatómicas sobre Tudorella ferruginea (Lamarck, 1822) (Mollusca, Prosobranchia, Pomatiasidae). Boletín de la Sociedad de Historia Natural de Baleares, 23, 69-78.

Ilano, A. S., Fujinaga, K., \& Nakao, S. (2003). Reproductive cycle and size at sexual maturity of the commercial whelk Buccinum isaotakii in Funka Bay, Hokkaido, Japan. Journal of the Marine Biological Association of the United Kingdom, 83(6), 1287-1294.

IUCN (2012). Red List Categories and Criteria: Version 3.1. ( $2^{\text {nd }}$ ed.). Gland, Switzerland and Cambridge, UK: IUCN

IUCN Standards and Petitions Working Group (2008). Guidelines for Using the IUCN Red List Categories and Criteria. Version 7. Standards and Petitions Working Group for the IUCN SSC Biodiversity Assessments SubCommittee in August 2008. Recuperado de http:// intranet.iucn.org/webfiles/doc/SSC/RedList/RedListGuidelines.pdf

Maceira, D. (2010). Variación estacional de la densidad poblacional, patrón de actividad y uso del hábitat de Caracolus sagemon (Mollusca: Camaenidae) en la Reserva Ecológica Siboney-Juticí, Cuba (Tesis de doctorado). Universidad de Alicante, España.

Maceira, D., \& Espinosa, J. (2016). Gastrópodos. En M. M. Hidalgo-Gato, J. Espinosa \& R. Rodríguez-León. (Eds.), Libro Rojo de Invertebrados Terrestres de Cuba (pp. 41-142). La Habana, Cuba: Editorial Academia.

Mateo, R. (1989). Paisajes 1. En Instituto de Geodesia de la Academia de Ciencias de Cuba y el Instituto Cubano de Geodesia y Cartografía (Eds.), Nuevo Atlas Nacional de Cuba (pp. XII.1.2-3.). España: Gráficas ALBER.

Moore, H. B. (1937). The biology of Littorina littorea. Part I. Growth of the shell and tissues, spawning, length of life and mortality. Journal of the Marine Biological Association of the United Kingdom, 21(2), 721-742.

ONEI (Oficina Nacional de Estadística e Información de la República de Cuba). (2017a). Anuario
Estadístico de Manzanillo 2016. Edición Octubre de 2017 (139pp). Recuperado de http://www.one.cu/ aed2016/33Granma/Municipios/06\%20Manzanillo. pdf

ONEI (Oficina Nacional de Estadística e Información de la República de Cuba). (2017b). Anuario Estadístico de Granma 2016. Edición Septiembre de 2017 (241pp). Recuperado de http://www.one.cu/ aed2016/33Granma/00\%20Granma.pdf

ONEI (Oficina Nacional de Estadística e Información de la República de Cuba). (2017c). Panorama Ambiental Cuba 2016. Centro de Gestión de la Información Económica, Medioambiental y Social. Edición Julio de 2017 (60 pp). Recuperado de http://www.one. $\mathrm{cu} /$ publicaciones/04industria/medioambientecifras/ medioamb2016.pdf

Pelseneer, P. (1926). La proportion relative des sexes chez les animaux et particulièrement chez les mollusques. Mémoires de l'Académie Royale de Belgique (Classe des Sciences), 8(11), 1-258.

Pfeiffer, L. (1858). Zur Molluskenfauna der Insel Cuba. Malakozoologische Blätter, 5, 37-49.

Sariego, O. R. (2006). Acercamiento a la definición del estado de conservación de Chondropoma neglectum (Gundlach in Pfeiffer; 1856) (Annulariidae: Mollusca). Revista Electrónica Granma Ciencia, 10(2), $1-14$.

Silva, A. R., \& Berovides, V. (1982). Acerca del concepto de nicho ecológico. Ciencias Biológicas, 8, 95-103.

Simone, L. R. (2004). Comparative morphology and phylogeny of representatives of the superfamilies of Architaenioglossans and the Annulariidae (Mollusca, Caenogastropoda). Arquivos do Museu Nacional de Rio de Janeiro, 62(4), 387-504.

Statsoft. (2015). STATISTICA. Statsoft Dell Inc. Version 13.0.

Tadeo, R. P., Alfonso, M. A., \& Berovides, V. (1999). Ecología de Zachrysia guanensis costulata (Gastropoda: Camaenidae) de la Sierra de San Carlos, Pinar del Río, Cuba. Revista Biología, 13(2-3), 95-102.

Thompson, F. G. (1978). A new genus of operculate land snails from Hispaniola with comments on the status of family Annulariidae. Nautilus, 92(1), 41-54.

Torre, C., \& Bartsch, P. (1938). The Cuban operculate land shells of the subfamily Chondropominae. Proceedings of the United States National Museum, 85, 193-425.

Watters, G. T. (2006). The Caribbean landsnail family Annulariidae. A revision of the higher taxa and catalog of the species. Leiden, The Netherlands: Backhuys Publishers. 